(C) 2020 IEEE. Personal use of this material is permitted. Permission from IEEE must be obtained for all other uses, in any current or future media, including reprinting/republishing this material for advertising or promotional purposes, creating new collective works, for resale or redistribution to servers or lists, or reuse of any copyrighted component of this work in other works. 


\section{The Marine Robotics Research Infrastructure Network (EUMarine Robots): An Overview}

\author{
Daniel Alcaraz \\ Oceanic Platform of the Canary Islands \\ Las Palmas, Spain \\ daniel.alcaraz@plocan.eu \\ Gerard Dooly \\ University of Limerick \\ Limerick, Italy \\ gerard.dooly@ul.ie \\ Martin Ludvigsen \\ Norwegian University of Science and \\ Technology \\ Trondheim, Norway \\ martin.ludvigsen@ntnu.no
António Pascoal
Instituto Superior Técnico
Lisbon, Portugal \\ antonio@isr.ist.utl.pt \\ Pere Ridao \\ University of Girona \\ Girona, Spain \\ pere@ silver.udg.es
}

\author{
Gianluca Antonelli \\ University of Genoa \\ Genova, Italy \\ antonelli@unicas.it
}

\author{
Niamh Flavin \\ Marine Institute \\ Galway, Ireland \\ niamh.flavin@Marine.ie \\ Jan Opderbecke \\ Institut Français de Recherche pour \\ l'exploitation de la Mer \\ Marseille, France \\ jan.Opderbecke@ifremer.fr
}

João Borges de Sousa

LSTS - University of Porto
Porto, Portugal
jtasso@fe.up.pt

Nikola Mišković

University of Zagreb

Zagreb, Croatia

nikola.miskovic@fer.hr

\author{
Massimo Caccia \\ Consiglio Nazionale delle Ricerche \\ Rome, Italy \\ massimo.caccia@cnr.it
}

\author{
Achim Kopf \\ University of Bremen \\ Bremen, Germany \\ akopf@marum.de
}

Matthew Palmer

Natural Environment Research Council Southampton, United Kingdom

rolm@noc.ac.uk

Roberto Petroccia

Centre for Maritime Research and

Experimentation

La Spezia SF, Italy

roberto.petroccia@cmre.nato.int

Sen Wang

Heriot-Watt University

Edinburgh, United Kingdom

s.wang@hw.ac.uk

The Marine robotics research infrastructure network (EUMarine Robots) addresses the H2020 call topic INFRAIA-022017: Integrating Activities for Starting Communities by mobilizing a comprehensive consortium of most of the key marine robotics research infrastructures which, in turn, mobilized stakeholders from different Member States, Associated Countries and other third countries to achieve the following main objective: open up key national and regional marine robotics research infrastructures (RIs) to all European researchers ensuring their optimal use and joint development to establish a world-class marine robotics integrated infrastructure.

EUMarine(EUMR) is organized around an evolving network model. It builds on existing networks of the EU marine robotics community and is synergistic with the worldwide marine robotics communities. EUMR is also aligned with the strategy for development of the European innovation ecosystem for robotics and autonomous systems, seeks to underpin the development of the next-generation observing systems, and complements large European Marine RIs. This paper is a quick overview of the main organizational components of EUMR: Transnational Access (TNA), Joint Research (JRA) and Networking Activities (NA). Please see the project webpage https://www.eumarinerobots.eu/ for complete details.

TNA aims to provide access to marine robotics infrastructures from the 15 EUMR partners. They include a large number of heterogeneous autonomous underwater, surface and air vehicles, ships and testbeds. There are two modalities of access to the general infrastructure in the form of access to marine robotics RIs operated by the partners and to collaborative sea experiments/use cases taking place in the Atlantic and Arctic oceans and in the Mediterranean. Access is granted through competitive calls. The first call closed October 1, 2018, with 26 eligible applications (70\% from Europe, 22 from Asia and $7 \%$ from North America), and 22 projects were approved. The second call closed August 15, 2019, with 31 applications and 28 projects approved. The third call opened in May 2020. A list of some of the TNA projects successfully completed includes: i) Investigation and experimental activities aimed at developing an AUV for Liquid-Argon Time Projection Chambers; ii) Detection And Mapping Of Submarine C02 Seeps; iii) Underwater Hyperspectral imaging - Scientific payload integration and evaluation on hybrid ROV; iv) Survey and Mapping of Falconera Cave; v) Integrated Software for Underwater Vehicle Interoperability; and vi) High-resolution 3D terrain models of cold- water coral Habitats in Blanes Canyon.

JRA addresses the development of advanced marine robotic systems and technologies with a view to substantially improve existing infrastructures and thus afford scientific and commercial end-users cutting edge tools for ocean exploration/exploitation. This is being actively pursued along all technical tasks: i) Advanced vision, acoustic, and manipulation systems for automated inspection and intervention operations; ii) Cooperative navigation and control of networked vehicles for increased autonomy at sea; iii) Hybrid acoustic-optical underwater communication networks for nextgeneration cooperative systems; iv) Advanced Systems for Under-Ice operations; v) Enhanced underwater LARS system; and, vi) Cooperative human-marine robot systems. JRA is also about taking these developments to sea and demonstrate operability and improvement of Technology Readiness level. Various ships were and will be made available for participation of EUMR partners demonstrating the potential of the developments accomplished in work packages on existing scientific cruises or on 
dedicated EUMR cruises. On the RV Polarstern cruise (PS119) in April/May 2019, some of our team members were participating in the evaluation and testing of the performance and limitations of the new telepresence capabilities on the German Research fleet. This was done by providing scientists and engineers with two-way satellite communication channels from shore to ship to ROV Quest on the seafloor. A second, smaller, opportunity was used on IFREMER's RV L'EUROPE in the Ligurian Sea off the coast of Nice to provide a testing scenario for optical underwater communication and visual surveying in a real environment. JRA developments were also demonstrated in the 2018 and 2018 editions of the Rapid Environmental Picture (REP) exercise that took place in Portugal.

Networking activities included co-organization and participation in the 2018 and 2019 editions of the Breaking the Surface interdisciplinary field workshop of marine robotics and applications (Croatia), the PLOCAN glider school (Spain), the EMRA annual conference, as well as the participation by FEUP, Portugal in the Exploring Fronts with Multiple Robots science cruise funded by the Schmidt Ocean Institute. Networking activities also include educational activities targeting the community at large. To meet these goals, an e-access infrastructure is also under development to provide access to electronic versions of training courses and tutorials, as well as to data sets from robotic experiments. EUMR is also working to maintain and further develop links within partners as well as international coordination groups such as the European Robotics Forum ERF, or the advanced community INFRAIA project Eurofleets+. Annual project workshops address research infrastructure coordination and development and provide a strategic vision of the critical aspects through SWOT analysis covering: i) standardization data, software, communication, and electrical interfaces; ii) Data management of vehicle and payload data, long-term policies for access and storage; iii) Open software policies; iv) Best practice in marine robot operation for the TNA programs, quality of service, risk identification and mitigation; and, v) Assessment of sites for testing and coherent guidelines for rules and procedures, local site constraints (safety requirements, access formalities, logistics etc.).

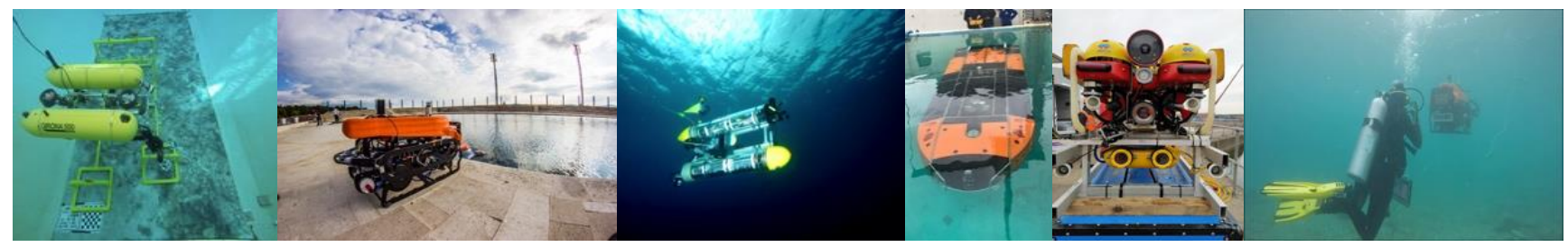

Fig. 1 A sample of the EUMarine Robots Network infrastructures

EUMR's vision it to foster and contribute to the development of methods, tools, and robot vehicles required for a sustained presence in the ocean by opening-up key national and regional marine robotics research infrastructures (RIs) to all European researchers and establishing a model of world-class marine robotics integrated infrastructure. Access to marine robotic infrastructures is key to a sustained presence in the ocean that will enable us to understand and monitor how key issues such as climate change, ocean acidification, unsustainable fishing, pollution, waste, loss of habitats and biodiversity, shipping, security, and mining are affecting global ocean sustainability and stewardship. Joint research activities towards integrated marine robotics research infrastructures, with strong emphasis on open innovation and open science, will be a stepping-stone for other developments worldwide. EUMR network activities are based on concept of evolving a scalable network model to become a world-class marine robotics network. In summary, the time is ripe for EUMR to have a lasting impact, well beyond the duration of the project.

Acknowledgements: The EUMarineRobots project - "Marine robotics research infrastructure network", is funded by the European Union's Horizon 2020 research and innovation programme under grant agreement No 731103. This support is gratefully acknowledged. 